\title{
Transformative Effects of Higher Magnetic Field in Fourier Transform Ion Cyclotron Resonance Mass Spectrometry
}

\author{
N. Murat Karabacak, ${ }^{\text {a }}$ Michael L. Easterling, ${ }^{\text {b }}$ Nathalie Y. R. Agar, ${ }^{c}$ and \\ Jeffrey N. Agar \\ a Department of Chemistry and Volen Center for Complex Systems, Brandeis University, Waltham, MA, USA \\ b Bruker Daltonics, Billerica, MA, USA \\ ' Department of Neurosurgery, Brigham and Women's Hospital, Harvard Medical School, Boston, MA, USA
}

\begin{abstract}
The relationship of magnetic field strength and Fourier transform ion cyclotron resonance mass spectrometry performance was tested using three instruments with the same design but different fields of 4.7, 7, and 9.4 tesla. We found that the theoretically predicted "transformative" effects of magnetic field are indeed observed experimentally. The most striking effects were that mass accuracy demonstrated $\sim$ second to third order improvement with the magnetic field, depending upon the charge state of the analyte, and that peak splitting, which prohibited automated data analysis at 4.7 T, was not observed at 9.4 T. (J Am Soc Mass Spectrom 2010, 21, 1218-1222) (c) 2010 American Society for Mass Spectrometry
\end{abstract}

$\mathrm{F}$ ourier transform ion cyclotron resonance mass spectrometry [1] (FT-ICR MS) is currently the highest resolution mass spectrometry method. The cyclotron frequency of ions in a given magnetic field (eq 1) gives a very accurate measure of the mass to charge ratio of ions.

$$
\omega=\frac{q B}{m}
$$

Numerous performance parameters in FT-ICR MS are predicted to improve with magnetic field, including linear improvements in mass resolving power and acquisition speed, and higher order improvements in mass accuracy, dynamic range, kinetic energy, and peak coalescence [2-7]. All of these parameters combine to determine the figure of merit of a mass analyzer, but not in a manner that is easily predicable. Improving FT-ICR MS performance is critical in many fields such as proteomics, petroleomics, and MALDI imaging, and in many cases enables, rather than improves, analytical capabilities.

In practice, however, a component with no a priori field dependence, for example field homogeneity [8] vacuum strength, or acquisition speed, which set fundamental limits on resolution [2], or a phenomenon with a convoluted electrical and magnetic field dependence such as peak coalescence [9] or phase locking [7, 10-14], can become the limiting factor and the determinant of performance. In addition, the relationship of

Address reprint requests to Dr. J. N. Agar, Department of Chemistry, Brandeis University, MS015, 415 South Street, Waltham, MA 02454, USA. E-mail: agar@brandeis.edu important instrument performance metrics can be affected by factors with no- or convoluted field dependences. For example, the relationship between mass accuracy and resolution is blurred by anything that affects ideal peak shapes [15-17].

There are isolated literature examples of what Marshall terms the "transformative effects" of higher magnetic fields. For example, post processing of $14.7 \mathrm{~T}$ FTMS spectrum to simulate a $7 \mathrm{~T}$ FTMS spectrum revealed that three compounds that were identifiable at 14.7 $\mathrm{T}$ appeared to be a single compound at $7 \mathrm{~T}$ [18]. In another example, the instrument's sensitivity for a complex and highly charged sample $(29 \mathrm{kDa}$ protein dissociation products) significantly increased when going from 6 T to 9.4 T FT-ICR [19]. On the other hand, despite the availability of $15 \mathrm{~T}$ instruments, the highest published resolving powers of $\sim 8,000,000(\mathrm{~m} / \mathrm{z} 1148)$ [20], $\sim 17,000,000(\mathrm{~m} / \mathrm{z} 1084.5)$ [21], $\sim 1,000,000(\mathrm{~m} / \mathrm{z}$ 12,360 ) [22] were acquired (in one case 11 years ago [20]) at $9.4 \mathrm{~T}$ or $7 \mathrm{~T}$, illustrating the importance of factors other than magnetic field. While the effects of field homogeneity upon instrument performance have been characterized [8], we are aware of no publication that systematically explores the relationship of FT-ICR MS performance and gross magnetic field. Here, we make an empirical determination of the merit of higher magnetic field, using the same instrument type, the same acquisition and processing software and methods, the same methods for optimization, the same user, and employing magnetic fields from 4.7 to $9.4 \mathrm{~T}$. Moreover, we perform a meta-analysis of reported mass accuracies of FTMS instruments ranging from 1 to $15 \mathrm{~T}$, which is consistent with our experimental findings. 


\section{Methods}

All chemicals except ubiquitin (Boston Biochem, Cambridge, MA, USA) were purchased from Sigma-Aldrich (St. Louis, MO, USA).

This study involved three separate commercial hybrid q-FT-ICR mass spectrometers (Bruker Apex-qe) with the same cell infinity ion trap geometry [23], electrospray source (Apollo 2), ion transfer optics, vacuum elements (with readings of below $4 \times 10^{-10} \mathrm{mbar}$ in the ICR cell during this study) but with different magnetic fields. A sodium formate (Sigma) $0.01 \mathrm{mg} / \mathrm{mL}$ solution was prepared in $50 \%$ acetonitrile $0.1 \%$ formic acid. A complex peptide and protein mixture with a concentration ladder spanning four orders of magnitude was prepared in $50 \%$ acetonitrile $0.1 \%$ formic acid and introduced by a syringe pump at $2 \mu \mathrm{L} / \mathrm{min}$. This mixture consisted of Substance P $(1 \mu \mathrm{M})$, insulin $(0.1$ $\mu \mathrm{M})$, orexin $\mathrm{B}(0.01 \mu \mathrm{M})$, ubiquitin $(0.1 \mu \mathrm{M})$, angioten$\sin (0.1 \mathrm{nM})$, myoglobin $(1 \mu \mathrm{M})$, human $\mathrm{Cu} / \mathrm{Zn}$ superoxide dismutase $(0.05 \mu \mathrm{M})$, ribonuclease $\mathrm{A}(0.5 \mu \mathrm{M})$ and lysozyme $(0.01 \mu \mathrm{M})$. One $\mu \mathrm{M}$ Substance $\mathrm{P}$ was used for ultra-high resolution narrowband experiment.

Instruments were tuned and operated using our routine techniques and parameters, which involved the following steps. Initially, electrospray source and transfer parameters were optimized for maximum signal magnitude. Static trapping [24] at relatively low trapping potentials $(1.0 \mathrm{~V} / 0.8 \mathrm{~V}$ for $4.7 \mathrm{~T}, 0.9 \mathrm{~V} / 0.8 \mathrm{~V}$ for 7.0 $\mathrm{T}$ and $1.2 \mathrm{~V} / 1.2 \mathrm{~V}$ for $9.4 \mathrm{~T}$ for front/rear trapping plates) was used. These trapping potentials were $\sim 30 \%$ greater than potential where no signal was observed (and presumably little trapping occurred). Next, excitation amplitude was "tuned" for each instrument by determining the excitation amplitude that yielded maximum mass accuracy when internal calibration was performed using sodium formate clusters. Resulting amplitudes were $1.25 \mathrm{~dB}$ for $9.4 \mathrm{~T}, 4.5 \mathrm{~dB}$ for $7.0 \mathrm{~T}$ and $9.75 \mathrm{~dB}$ for $4.7 \mathrm{~T}$. Using these optimized parameters, spectra of the peptide and protein mixture were acquired. Ions were directly infused into an electrospray source at $2 \mu \mathrm{L} / \mathrm{min}$ flow rate, externally accumulated in the source and collision cell hexapole for $1 \mathrm{~s}$ each, and transferred to the ICR cell using ion transfer optics that had been previously optimized for signal intensity. Chirp excitation and image charge detection was performed. Three, 2-M-word datasets in the range of $\mathrm{m} / \mathrm{z}$ 274-3000 were acquired and 4,16, or 64 scans were averaged for each dataset. The FID was multiplied by a sine bell apodization function and was Fourier transformed. The narrowband experiment (Figure 3) involved a 32-k-word dataset covering 3.0 Thomson (Th) and was averaged 325 times.

We tried to eliminate bias during data analysis in the following way. Only the peaks (1) with $\mathrm{S} / \mathrm{N}$ greater than 10, and (2) that were commonly found in each spectrum (4.7, 7.0 and 9.4 T) were used for automatic, internal, second order calibration with the search cutoff of 0.03 Th (Bruker DataAnalysis software V. 3.4, for- mula is Ledford-Rempel-Gross type [25]). We used eight peaks for our complex protein/peptide mixture and 15 peaks for sodium formate in the $\mathrm{m} / \mathrm{z}$ range 400 to 1600. Weighted average and standard deviations (times two) of three spectra were plotted for the mixture. The theoretical masses were calculated by software that was developed in our lab and published elsewhere [26].

A meta analysis of small molecule $(<4000 \mathrm{Da})$ literature was undertaken by taking mass accuracy values from manuscripts that reported mass accuracy and analyzed multiple analytes. Irrespective of how mass accuracies were reported in the original articles, we reported their mass accuracies as absolute average mass error. To eliminate bias, any manuscript that was found to fit these criteria was used and no data were eliminated as "outliers." Thus, the results shown were not "cherry picked" for studies that fit our hypothesis.

\section{Results and Discussion}

\section{Peak Shapes and Splitting}

The transition from 4.7 to $9.4 \mathrm{~T}$ magnetic field (Figures $1,2)$ was transformative, not incremental. Spectra consisting of 2000 peaks (due to peak splitting) at $4.7 \mathrm{~T}$ were not amenable to automated, nor often manual, interpretation (Figure 1), and rendered concepts such as resolution meaningless (peak splitting results in uncertainty in where the signal of a given molecular ion begins and ends). In contrast to the $4.7 \mathrm{~T}$ results, the spectrum of the same analyte (Figure 1) at $9.4 \mathrm{~T}$ had 200 peaks and could be correctly processed by a computer. Thus, an instrument with half the magnetic field yielded $\sim 10$ times the number of false positive peaks. The 4.7 to $9.4 \mathrm{~T}$ transition also led to a second-to-third order improvement in mass accuracy (vide infra), which taken together with $\sim 10$-fold decreased in false positive peak assignment, could lead to up to a 40-fold decrease in the number of false positive identifications using an automated database search (at a constant $\mathrm{S} / \mathrm{N}$ threshold).

\section{Resolution}

Quantification of resolution requires an ideal peak shape that was not observed in this work until a field of $9.4 \mathrm{~T}$ (Figure 1). Indeed, as a result of the high $\mathrm{S} / \mathrm{N}$ of the artifactual sidebands, a mathematical definition of resolution (i.e., automatable definitions based upon peak shapes or line derivatives) for the 4.7 and $7 \mathrm{~T}$ instruments would be exceedingly difficult to establish and probably require empirically defined limits to prevent grossly overestimating the resolution. The same artifactual sidebands also precluded the quantification of peak coalescence. In other words, only at a field of 9.4 $\mathrm{T}$ (and presumably above this) could we provide a meaningful estimate of instrument resolution. 


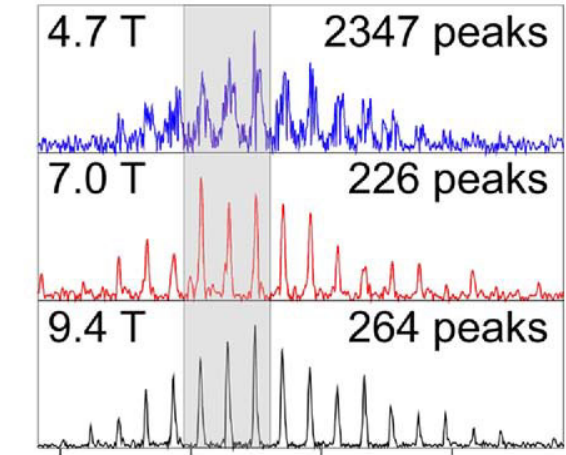

1140.61141 .01141 .41141 .8
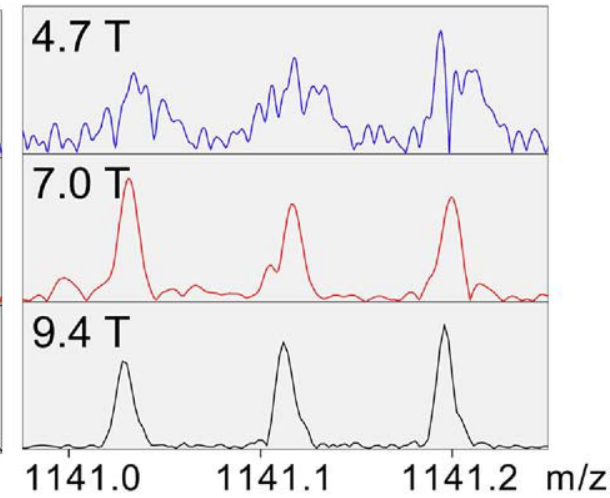

Figure 1. The relationship of magnetic field and peak shape using Bruker FT-ICR MS instruments. Shown are isotopomer distributions of ribonuclease A. Only at $9.4 \mathrm{~T}$ does artifactual peak splitting end. Numbers of peaks indicated are automatically calculated for the entire $\mathrm{m} / \mathrm{z}$ range, using DataAnalysis software (Bruker Daltonics) using a 10\% base peak magnitude threshold and FTMS as the instrument type.

\section{Mass Measurement Accuracy}

In Figure 2a, average (absolute) deviations in mass were plotted when internal, second-order calibration was performed. The mass accuracy of a small analyte (sodium formate clusters), and of our peptide and protein mixture, which have higher charge and mass on average, increased as the second and third order of the magnetic field, respectively (Figure 2, right panel). We are not aware of theoretically or empirically-derived predictions of such third order effects and are unsure of their mechanistic origin. We note empirically that the slope of the free induction decay (and presumably the rate of dephasing) tends to be approximately first-order for small, singly charged molecules, but is higher order for larger, multiply charged ions. Thus, a putative (and qualitative) explanation for the observed third order effects is increased space charge-related ion dephasing.

When our internally calibrated experimental data in Figure $2 \mathrm{a}$ is extrapolated to a magnetic field of $15 \mathrm{~T}$, the average predicted mass error for small molecules is 43 $\mathrm{ppb}$. This value is close to the $34 \mathrm{ppb}$ RMS error value achieved with a $14.7 \mathrm{~T}$ instrument using external calibration [18]. This extrapolation also predicts $22 \mathrm{ppb}$ mass accuracy for $21 \mathrm{~T}$ magnetic field instruments, which are currently in development [27]. On the other hand, the current range of performance of $\sim 15 \mathrm{~T}[18$, $28]$ instruments are an indication that such improvement must not be taken as a given. Although mag-

\section{Current work (int. cal.)}
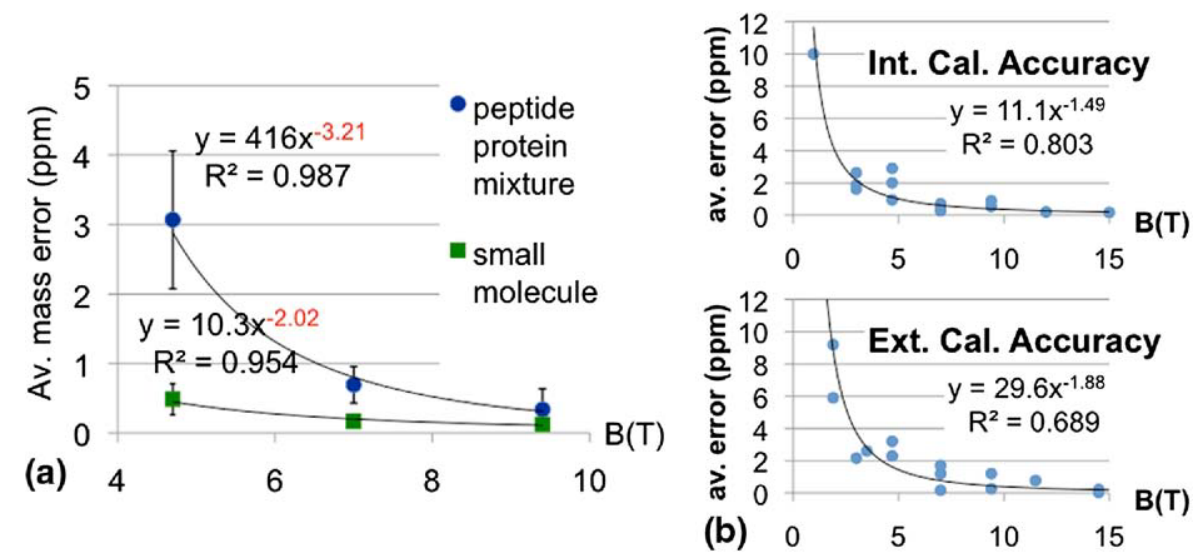

Figure 2. The relationship of magnetic field and accuracy. (a) Arithmetic mean of absolute mass measurement error using Bruker FT-ICR mass spectrometers (left panel). Internal calibration of NaFA and a complex mixture of nine proteins and peptides were compared with their theoretical values. Accuracy improves $\sim$ second order in magnetic field for NaFA sample and $\sim$ third order for complex protein and peptide mixture. Extrapolation to $15 \mathrm{~T}$ predicts $22 \mathrm{ppb}$ mass error. The curves are an average of three spectra and shown error bars represent two standard deviations each direction. Error bars for small molecule data at 7 and $9.4 \mathrm{~T}$ were too small for Excel to draw. (b) Magnetic field's effect on accuracy in literature. Experimental average accuracies from 24 references were plotted with respect to the magnetic field used. Values are from analyses that used different analytes, instruments, users, etc. A detailed table of the references is given as Supplementary Table. 


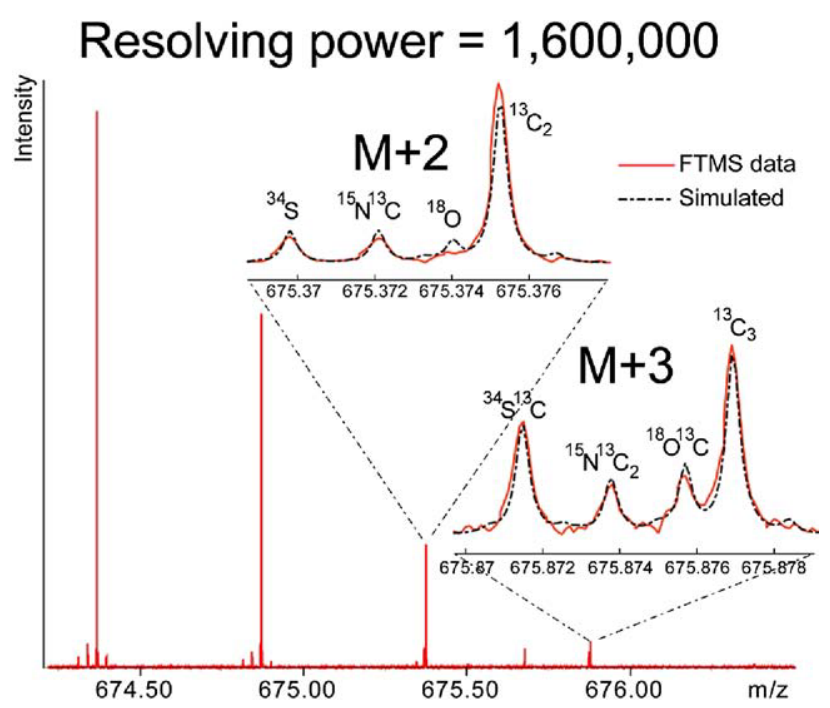

Figure 3. FT-ICR MS with 9.4 Tesla magnetic field detects isotopic fine structure of Substance P. The simulation of Substance $P$ mass spectrum at 1,600,000 resolution fits the data.

netic field was the factor limiting accuracy and resolution in the range of magnetic fields used here 4.7-9.4 T, other factors may turn out to limit accuracy and resolution at higher fields. For example, ion kinetic energies increase as the second-order of the magnetic field, resulting in a concomitant increase in unwanted fragmentation, and requiring improved vacuum systems [29].

This study analyzed two mixtures of molecules spanning a wide range of size and charge. Internal calibration was used to eliminate some of the variation in accuracy over time (due to, for example, electric or magnetic field drift). A meta analysis of over 20 studies reporting FTMS mass accuracy spanning field strengths of 1-15 T revealed roughly second-order improvements of mass accuracy with magnetic field (field's power of 1.5 and 1.9 for internal and external calibration respectively) consistent with our finding of second-order (Figure $2 \mathrm{~b}$ and Supplementary Table, which can be found in the electronic version of this article). These studies analyzed small molecules and purified peptides, and as a result are best compared with our small molecule mixture.

\section{Isotopic Fine Structure}

There are currently only a handful of groups who have reported the detection of isotopic fine structure using an FTMS. Figure 3, for example, demonstrates a mass resolution of 1.6 million at $\mathrm{m} / z 675$ at $9.4 \mathrm{~T}$ magnetic field. Another transformative aspect of the 4.7 to $9.4 \mathrm{~T}$ transition was therefore the facile detection of the isotopic fine structure, which provides a window into the molecular composition (e.g., counting the number of sulfur atoms) of a molecular ion (Figure 3), of molecules as large as $\sim 5000 \mathrm{Da}$. For example, using a commercially available mass spectrometer and stan- dard data processing routines we can detect isotopic fine structure of insulin at a $9.4 \mathrm{~T}$ field (data not shown). Detection of the isotopic fine structures of molecules up to $16 \mathrm{kDa}$ was achieved by Marshall and colleagues [20] and required (among other things) a custom-built instrument with a $10 \mathrm{~cm}$ ion trap diameter, manual, frequency-domain signal averaging, and heavy isotope depleted samples. Due to the peak splitting observed at lower magnetic fields, this study could not further quantify the relationship of resolution (nor peak coalescence) and magnetic field. The detection of isotopic fine structure will be facilitated by increased resolution, which has a predicted first-order increase with magnetic field, and by decreased peak coalescence, which has a predicted second-order decrease with magnetic field. It will therefore be interesting to see how the upper mass limit for the detection of isotopic fine structure increases in the transition from $9.4 \mathrm{~T}$ to even higher fields. With increased mass accuracy and isotopic fine structure, the upper mass limit for determining the molecular formula corresponding to a given peak would increase immensely, allowing the molecular formulae (and often the identity) of many molecular ions to be determined [30]. This increased resolution and accuracy will benefit from recently developed software that can efficiently calculate theoretical spectra and isotopic fine structure [26] of large molecules, including proteins.

\section{Conclusions}

Using similar mass spectrometers at $4.7,7$, and $9.4 \mathrm{~T}$, this study demonstrates second and third order improvements in mass accuracy with increasing magnetic field for small singly charged, and large multiply charged analytes, respectively. A meta-analysis of the existing literature spanning the range of $1-15 \mathrm{~T}$ was also consistent with a higher order relationship between magnetic field and mass measurement accuracy. In addition to the improvement of mass accuracy, peak splitting also significantly decreased and peak shapes correspondingly improved with increasing magnetic field.

\section{Acknowledgments}

The authors acknowledge support for this work by National Institutes of Health grant R01NS065263 to JNA, and from the Brain Science Foundation and the Daniel E. Ponton Fund for the Neurosciences to NYRA.

\section{Appendix A Supplementary Material}

Supplementary material associated with this article may be found in the online version at doi:10.1016/ j.jasms.2010.03.033. 


\section{References}

1. Comisarow, M. B.; Marshall, A. G. Fourier-Transform Ion-Cyclotron Resonance Spectroscopy. Chem. Phys. Lett. 1974, 25, 282-283.

2. Comisarow, M. B.; Marshall, A. G. Theory of Fourier-Transform IonCyclotron Resonance Mass-Spectroscopy 1. Fundamental Equations and Low-Pressure Line-Shape. J. Chem. Phys. 1976, 64, 110-119.

3. Marshall, A. G. Theoretical Signal-to-Noise Ratio and Mass Resolution in Fourier-Transform Ion-Cyclotron Resonance Mass-Spectrometry. Anal. Chem. 1979, 51, 1710-1714.

4. Marshall, A. G.; Guan, S. Advantages of High Magnetic Field for Fourier Transform Ion Cyclotron Resonance Mass Spectrometry. Rapid Commun. Mass Spectrom. 1996, 10, 1819-1823.

5. Marshall, A. G.; Hendrickson, C. L.; Jackson, G. S. Fourier Transform Ion Cyclotron Resonance Mass Spectrometry: A primer. Mass Spectrom. Rev. 1998, 17, 1-35.

6. Mitchell, D. W.; Smith, R. D. Cyclotron Motion of Two Coulombically Interacting Ion Clouds with Implications to Fourier-Transform Ion Cyclotron Resonance Mass Spectrometry. Phys. Rev. E 1995, 52, 21.

7. Mitchell, D. W.; Smith, R. D. Prediction of a Space Charge Induced Upper Molecular Mass Limit Towards Achieving Unit Mass Resolution in Fourier Transform Ion Cyclotron Resonance Mass Spectrometry. J. Mass Spectrom. 1996, 31, 771-790.

8. Laukien, F. H. The Effects of Residual Spatial Magnetic-Field Gradients on Fourier-Transform Ion-Cyclotron Resonance-Spectra. Int. I. Mass Spectrom. Ion Processes 1986, 73, 81-107.

9. Boldin, I. A.; Nikolaev, E. N. Theory of Peak Coalescence in Fourier Transform Ion Cyclotron Resonance Mass Spectrometry. Rapid Commun. Mass Spectrom. 2009, 23, 3213-3219.

10. Jeffries, J. E.; Barlow, S. E.; Dunn, G. H. Theory of Space-Charge Shift of Ion cyclotron resonance frequencies. Int. J. Mass Spectrom. Ion. Processes 1983, 54, 169-187.

11. Nikolaev, E. N.; Heeren, R. M. A.; Popov, A. M.; Pozdneev, A. V.; Chingin, K. S. Realistic Modeling of Ion Cloud Motion in a Fourier Transform Ion Cyclotron Resonance Cell by Use of a Particle-in-Cell Approach. Rapid Commun. Mass Spectrom. 2007, 21, 3527-3546.

12. Wong, R. L.; Amster, I. J. Experimental Evidence for Space-Charge Effects Between Ions of the Same Mass-to-Charge in Fourier-Transform Ion Cyclotron Resonance Mass Spectrometry. Int. J. Mass Spectrom. 2007, 265, 99-105.

13. Hendrickson, C. L.; Beu, S. C.; Laude, D. A. Two-Dimensional CoulombInduced Frequency Modulation in Fourier Transform Ion Cyclotron Resonance: A Mechanism for Line Broadening at High Mass and for Large Ion Populations. J. Am. Soc. Mass Spectrom. 2008, 8, 906-916.

14. Mitchell, D. W.; Smith, R. D. Cyclotron Motion of Two Coulombically Interacting Ion Clouds with Implications to Fourier-Transform Ion Cyclotron Resonance Mass Spectrometry. Phys. Rev. E 2008, 21, $4366-$ 4386.

15. White, R. L.; Ledford, E. B.; Ghaderi, S.; Wilkins, C. L.; Gross, M. L. Resolution and Signal-to-Noise in Fourier-Transform Mass-Spectrometry. Anal. Chem. 1980, 52, 1525-1527.
16. Marshall, A. G.; Comisarow, M. B.; Parisod, G. Relaxation and Spectral Line Shape in Fourier Transform Ion Resonance Spectroscopy. J. Chem. Phys. 1979, 71, 4434.

17. Ling, C.; Cottrell, C. E.; Marshall, A. G. Effect of Signal-to-Noise Ratio and Number of Data Points Upon Precision in Measurement of Peak Amplitude, Position, and Width in Fourier-Transform Spectrometry. Chemomet. Intelligent Lab. Syst. 1986, 1, 51-58.

18. Schaub, T. M.; Hendrickson, C. L.; Horning, S.; Quinn, J. P.; Senko, M. W.; Marshall, A. G. High-Performance Mass Spectrometry: Fourier Transform Ion Cyclotron Resonance at 14.5 Tesla. Anal. Chem. 2008, 80, 3985-90.

19. Senko, M. W.; Hendrickson, C. L.; PasaTolic, L.; Marto, J. A.; White, F. M.; Guan, S. H.; Marshall, A. G. Electrospray Ionization Fourier Transform Ion Cyclotron Resonance at 9.4 T. Rapid Commun. Mass Spectrom. 1996, 10, 1824-1828.

20. Shi, S. D.; Hendrickson, C. L.; Marshall, A. G. Counting Individual Sulfur Atoms in a Protein by Ultrahigh-Resolution Fourier Transform Ion Cyclotron Resonance Mass Spectrometry: Experimental Resolution of Isotopic Fine Structure in Proteins. Proc. Natl. Acad. Sci. U.S.A. 1998 95, 11532-11537.

21. Brustkern, A. M. Rempel, D. L.; Gross. M. L. An Electrically Compensated Trap Designed to Eighth Order for FT-ICR Mass Spectrometry. J. Am. Soc. Mass Spectrom. 2008, 19, 1281-1285.

22. Wilkins, C. L. Impressive Developments in Mass Spectrometry. TrAC Trends Anal. Chem. 2007, 26, 65-67.

23. Caravatti, P.; Allemann, M. The Infinity Cell-a New Trapped-Ion Cell with Radiofrequency Covered Trapping Electrodes for Fourier-Transform Ion Cyclotron Resonance Mass Spectrometry. Org. Mass Spectrom. 1991 26, 514-518.

24. Hofstadler, S. A.; Laude, D. A. Trapping and Detection of Ions Generated in a High Magnetic-Field Electrospray Ionization Fourier-Transform IonCyclotron Resonance Mass Spectrometer. J. Am. Soc. Mass Spectrom. 1992, 3, 615-623.

25. Ledford, E. B.; Rempel, D.; Gross, M. L. Space-Charge Effects in Fourier-Transform Mass Spectrometry-Mass Calibration. Anal. Chem. $1984,56,2744-2748$.

26. Li, L.; Kresh, J. A.; Karabacak, N. M.; Cobb, J. S.; Agar, J. N.; Hong, P. A Hierarchical Algorithm for Calculating the Isotopic Fine Structures of Molecules. J. Am. Soc. Mass Spectrom. 2008, 19, 1867-1874.

27. Marshall, A. G.; Hendrickson, C. L.; Ernmetta, M. R.; Rodgers, P. Blakney, G. T.; Nilsson, C. L. Fourier Transform Ion Cyclotron Resonance: State of the Art. Eur. J. Mass Spectrom. 2007, 13, 57-59.

28. Palmblad, M.; Drijfhout, J. W.; Deelder, A. M. High Resolution Mass Spectrometry for Rapid Characterization of Combinatorial Peptide Libraries. J. Combinat. Chem. 2010, 12, 65-68.

29. Tolmachev, A.; Robinson, E. W.; Wu, S.; Pasa-Tolic, L.; Smith, R. D. FT-ICR MS Optimization for the Analysis of Intact Proteins. Int. J. Mass Spectrom. 2009, 287, 32-38.

30. Software for extracting accurate mass from FT-ICR MS data is commercially available from "Composer" (Sierra Analytics, Inc., http://www. MassSpec.com). 